\title{
Long-term follow-up of patients with insomnia
}

\author{
Leon D. Rosenthal, MD, Diana C. Dolan, BS, Daniel J. Taylor, PhD, and Emily Grieser, BS
}

It has been suggested that patients with insomnia who are lost to followup continue to experience symptoms. From winter 2006 to spring 2007, we contacted consecutive patients initially seen at a multidisciplinary sleep medicine clinic 3 to 5 years earlier and asked them about continuing symptoms. Among the 58 patients who agreed to participate, 43 (74\%) reported that they still experienced difficulty initiating and/or maintaining sleep. Thirty-one of those patients had continued to receive treatment, 11 from a sleep medicine specialist and the remainder from other physicians. The majority listed zolpidem or eszopiclone as their primary medication, and many took multiple medications, including over-the-counter drugs and alternative medications. Most patients with insomnia were unaware of cognitive-behavioral therapy. These findings emphasize the need for primary care physicians to monitor symptoms of insomnia and to remain vigilant about potential treatment complications.

nsomnia represents a frequent sleep-related complaint that has been recognized as a "significant economic burden" due to its effects on productivity and medical expenditures (1). Insomnia costs employers an estimated 4.4 days of wages per untreated individual over a period of 6 months. In addition, patients with insomnia incur significantly higher medical expenses than people without insomnia. While up to one third of the population has insomnia at some point in their lives, roughly $10 \%$ of the US population has chronic insomnia. Thus, the consequences of this chronic condition are substantial. Yet, there is a paucity of clinical data on the long-term progression of the condition. This study evaluates the long-term symptomatic status of patients with a chief complaint of insomnia.

\section{METHODS}

Consecutive patients initially seen at a multidisciplinary sleep medicine clinic were contacted by telephone from winter 2006 to spring 2007, 3 to 5 years after initial patient consultation. Calls were attempted twice to all listed numbers, and patients agreeing to participate were asked about the status of their insomnia-related symptoms. Statistical analyses were completed with JMP (Version 7, SAS Institute Inc., Cary, $\mathrm{NC}$ ). Institutional review board approval was obtained for the study.

\section{RESULTS}

Of 155 patients with contact information available, $100 \mathrm{had}$ active telephone numbers. Of those, 61 answered the phone on the first or second attempt, 58 completed at least a part of the study, and 52 answered all questions in the interview. A response rate of $58 \%$ was achieved among patients who were still potentially accessible.

There were 22 men and 36 women with an average age of 50 \pm 15 years and an average body mass index of $25 \pm 5 \mathrm{~kg} / \mathrm{m}^{2}$. The 58 patients who participated at least partially in the survey were not statistically different from the 42 who did not participate in terms of demographic characteristics, symptom duration at initial consultation, Epworth Sleepiness Scale scores, and subjective sleep parameters, with the exception that participants were older $(50 \pm 15$ vs $40 \pm 15 ; P=0.02)$.

The majority of the patients, 43 (74\%), still experienced difficulty initiating and/or maintaining sleep, and $67 \%$ of those who were symptomatic reported both symptoms. Of the 43 with symptoms, $31(72 \%)$ reported they currently received treatment, $11(26 \%)$ did not, and 1 person declined to answer. Overall, $53 \%$ of initially seen patients who responded still required treatment for insomnia several years later.

For the 31 patients who received treatment currently, 11 saw a sleep medicine physician, 11 saw their primary care physician, 3 saw an obstetrician/gynecologist, 3 saw a psychiatrist, 1 saw an allergist, and 2 patients declined to identify provider information. Thus, 55\% of patients in long-term treatment were followed by their primary care physician or psychiatrist.

In terms of medication, 31 (53\%) patients (not necessarily fully overlapping with those still considered in treatment) took prescription medication; $42 \%$ of those took more than one medication. The majority listed zolpidem or eszopiclone as their primary medication (Table). Patients taking two prescription medications for sleep primarily received treatment from either a sleep medicine physician $(4 / 11 ; 36 \%)$ or a primary

From Sleep Medicine Associates of Texas, Dallas, Texas (Rosenthal, Dolan), and the Department of Psychology, The University of North Texas, Denton, Texas (Dolan, Taylor, Grieser).

Corresponding author: Leon D. Rosenthal, MD, Sleep Medicine Associates of Texas, 4708 Alliance Boulevard, Suite 725, Plano, Texas 75093 (e-mail: Idr@ sleepmed.com). 
Table. Medications for insomnia used at 3- to 5-year follow-up

\begin{tabular}{lcc}
\hline Medication & First $(\mathbf{n}=\mathbf{3 1})$ & Second $(\mathbf{n}=\mathbf{1 3})$ \\
\hline Zolpidem/eszopiclone & 19 & 3 \\
Benzodiazepines & 4 & 5 \\
Gabapentin & 1 & 0 \\
Antidepressants & 4 & 4 \\
Gamma-hydroxybutyric acid & 1 & 0 \\
Ramelteon & 1 & 0 \\
Muscle relaxant & 1 & 0 \\
Antipsychotic & 0 & 1 \\
\hline
\end{tabular}

care physician $(4 / 11 ; 36 \%)$; note that two patients declined to identify provider type. Some patients who took prescription medication also took over-the-counter medication $(4 / 31 ; 13 \%)$, and some patients used alternative medicines, such as herbal supplements $(5 / 58$ [8\%] of the total and $2 / 31$ [6\%] of those taking prescription medication).

Patients were asked if they had heard of cognitive-behavioral therapy for insomnia; $67 \%$ were unaware of the availability of a nonpharmacological treatment for insomnia such as cognitive-behavioral therapy.

\section{DISCUSSION}

A large number of patients initially seen in a sleep medicine clinic continue to experience insomnia years after seeking initial treatment, with over half involved in long-term treatment and requiring medication. Additionally, many take multiple medications, including over-the-counter drugs and alternative medications, which are not always reported to the treating physician. Most patients with insomnia reported distress about the continued need for medication and were unaware of cognitivebehavioral therapy, despite the growing availability and evidence of effectiveness of this therapeutic option (2).

Treatment of insomnia in the present cohort was frequently provided by primary care providers, with sleep medicine and psychiatric physicians only following a fraction of the patients. These findings emphasize the need for primary care physicians to monitor symptoms of insomnia and to remain vigilant about potential treatment complications. This issue is particularly relevant given the potential side effects of some of the currently prescribed hypnotics (3) and the increased reliance on chronic use of hypnotic medication. Furthermore, cognitive-behavioral therapy seems to be underutilized in this population, which would otherwise appear ideal for this type of intervention.

This study represents one of the few longitudinal studies among clinic patients with chronic insomnia. The chronic nature of insomnia was confirmed, and the continued reliance on psychopharmacologic intervention suggests the need for sustained clinical surveillance of this population.

1. Colten HR, Altevogt BM, eds. Sleep Disorders and Sleep Deprivation: An Unmet Public Health Problem. Washington, DC: National Academies Press, 2006.

2. Sivertsen B, Omvik S, Pallesen S, Bjorvatn B, Havik OE, Kvale G, Nielsen $\mathrm{GH}$, Nordhus IH. Cognitive behavioral therapy vs zopiclone for treatment of chronic primary insomnia in older adults: a randomized controlled trial. JAMA 2006;295(24):2851-2858.

3. Morgenthaler TI, Silber MH. Amnestic sleep-related eating disorder associated with zolpidem. Sleep Med 2002;3(4):323-327. 